Check for updates

Cite this: Mater. Chem. Front., 2020, 4, 1206

Received 9th January 2020

Accepted 14th February 2020

DOI: 10.1039/d0qm00012d

rsc.li/frontiers-materials

\title{
Aggregation-induced emission polymers for high performance PLEDs with low efficiency roll-off $\dagger$
}

\author{
Jiabao $\mathrm{Gu}^{\mathrm{a}}$ Zeng $\mathrm{Xu},{ }^{\mathrm{a}}$ Dongge Ma, (D) ${ }^{\mathrm{a}}$ Anjun Qin (D) *a and Ben Zhong Tang (D) ${ }^{\mathrm{ab}}$
}

As the congener of organic light-emitting diodes, polymeric light-emitting diodes (PLEDs) possess a number of distinct merits, such as a low-cost wet fabrication process, which make them applicable in large-area flexible display and lighting fields. However, most emissive polymers used in PLEDs suffer from the aggregation-caused quenching (ACQ) effect, which makes the device show large efficiency roll-off. In this work, two polymers, pTPE-DPA-Cz and pTPE-DPA-Flu, featuring aggregation-induced emission (AIE) characteristics were facilely synthesized through Suzuki-Miyaura polycoupling reaction by incorporating the AIE unit of TPE-DPA in their main chains. The resultant polymers possess good filmforming ability, excellent thermal stability and high photoluminescence quantum yields (PLQYs) in their film states, facilitating the fabrication of PLEDs through a solution process. Indeed, the PLEDs using PTPE-DPA-CZ and pTPE-DPA-Flu as emitting layers (EMLs) could achieve a maximum external quantum efficiency (EQE) of $3.26 \%$ (doped EML) and current efficiency of $3.69 \mathrm{~cd} \mathrm{~A}^{-1}$ (non-doped EML). Notably, all the devices exhibit a quite low efficiency roll-off. This work indicates that AIE polymers are ideal candidates for the construction of high performance PLEDs with low efficiency roll-off.

\section{Introduction}

Organic light-emitting diodes (OLEDs) have been widely applied in the fields of display and lighting since the first report by Tang and VanSlyke in 1987 because they possess a lot of distinctive merits such as excellent image quality, broad viewing angle, and being light, thin and flexible. ${ }^{1}$ In recent years, with the trend in OLED applications shifting to large-area lighting and flexible display fields, the low-cost wet fabrication process has attracted much attention, which is more economical than the dry process. ${ }^{2}$ In order to meet the requirements for wet fabrication processes, polymeric materials showing excellent filmforming ability were introduced into OLEDs, and therefore, extensive research on polymeric LEDs (PLEDs) has been conducted. ${ }^{3}$

The first PLED was reported in 1990, in which poly( $p$-phenylene vinylene)s were used as the emitter. However, the external quantum efficiency (EQE) was only $0.05 \%{ }^{4}$ Although high performance

\footnotetext{
${ }^{a}$ State Key Laboratory of Luminescent Materials and Devices, Guangdong Provincial Key Laboratory of Luminescence from Molecular Aggregates, Center for Aggregation-Induced Emission, South China University of Technology, Guangzhou 510640, China. E-mail: msqinaj@scut.edu.cn

${ }^{b}$ Department of Chemistry, Hong Kong Branch of Chinese National Engineering Research Centre for Tissue Restoration and Reconstruction, Institute for Advanced Study, and Department of Chemical and Biological Engineering, The Hong Kong University of Science \& Technology, Clear Water Bay, Kowloon, Hong Kong, China $\dagger$ Electronic supplementary information (ESI) available: Synthetic procedures, structure characterization, and thermal stability, lifetime of the polymers, and device performance. See DOI: 10.1039/d0qm00012d
}

PLEDs have been reported in recent years, the polymers used for the emitting layers (EMLs) mostly suffer from the aggregationcaused quenching (ACQ) effect, which greatly hampers the development of PLEDs. Therefore, to avoid the ACQ effect, the proportion of luminous groups in polymers for PLEDs is controlled at a low degree, typically lower than $20 \%$. Moreover, most of the PLEDs with ACQ polymers as EMLs encountered the problem of high efficiency roll-off, which further limits their applications. ${ }^{5}$

To construct practically applicable PLEDs, the following requirements should be fulfilled. First, the emissive polymers utilized as the EMLs of PLEDs should be easily synthesized with good solubility and high photoluminescence quantum yield (PLQY); second, the PLEDs should have high performance with low efficiency roll-off; ${ }^{6}$ third, the electroluminescent (EL) spectrum of the PLEDs should be stable. Thus, the polymers featuring aggregation-induced emission (AIE) characteristics should be ideal candidates for the construction of PLEDs.

AIE, conceptually coined by Tang et al. in 2001, refers to a unique phenomenon that a kind of non- or weakly emissive molecule is induced to emit intensely upon aggregation or being fabricated into thin films. AIE is exactly opposite to ACQ, thus, the AIE luminogens (AIEgens) have attracted much attention and a large variety of them with great structural diversity have been developed, such as tetraphenylethylene (TPE), hexaphenylsilole (HPS), distyreneanthracene (DSA), tetraphenylbenzene (TPB) ${ }^{7}$ and tetraphenylpyrazine (TPP), ${ }^{8}$ and their derivatives.

Compared with low mass AIEgens, AIE polymers, however, have been less explored. ${ }^{9}$ According to the restriction of intramolecular 
motion (RIM), the mechanism of AIE, the intramolecular motion of the AIE units in polymers is more easily restricted than in low mass molecules, leading to a stronger fluorescence in the aggregate and/or film state. ${ }^{10}$ Furthermore, AIE polymers enjoy many outstanding features such as good processability, facile functionalization, structural diversity and high thermal stability, which enable them to be applied in diverse areas. ${ }^{11-14}$

However, the application of AIE polymers in PLEDs is rarely reported. In 2012, Li et al. constructed linear and hyperbranched AIE polymers bearing the AIE unit of TPE and they were used to fabricate PLEDs as non-doped EMLs. ${ }^{12}$ The devices showed a current efficiency (CE) in the range of 0.093 to $2.13 \mathrm{~cd} \mathrm{~A}^{-1}$. In 2018 , they further synthesized hyperbranched polymers based on tetraphenylcyclopentadiene and fabricated PLEDs using them as doped EMLs but with high efficiency rolloff. ${ }^{13}$ Thus, PLEDs with higher performance and low efficiency roll-off with AIE polymers as EMLs should be further developed.

In this work, we synthesized two TPE-based AIE polymers, pTPE-DPA-Cz and pTPE-DPA-Flu, through a Suzuki-Miyaura polycoupling reaction (Scheme 1). In addition to the excellent thermal stability, the resultant polymers exhibit PLQYs up to $63.3 \%$ in the doped thin films. By utilizing these AIE polymers as EMLs, PLEDs were facilely fabricated via a solution-process. The results show that the device with 5\% pTPE-DPA-Flu in a 4,4'-di(9H-carbazol-9-yl)-1,1'-biphenyl (CBP) host as EML exhibits a maximum EQE of $3.26 \%$ and the non-doped device using PTPE-DPA-Cz as the EML could perform with $\mathrm{CE}$ as high as $3.69 \mathrm{~cd} \mathrm{~A}^{-1}$. More excitingly, low efficiency roll-off of the devices was realized, providing an effective molecular design strategy for AIE polymer based PLEDs.

\section{Results and discussion}

\section{Synthesis}

The synthetic routes to the monomers are shown in Scheme S1 (ESI $\dagger$ ) and those to the AIE polymers pTPE-DPA-Cz and pTPEDPA-Flu are presented in Scheme 1. The Suzuki-Miyaura polycoupling of the AIE unit containing dibromides and diborates bearing carbazole and fluorene moieties in the presence of the catalytic system of $\mathrm{Pd}\left(\mathrm{PPh}_{3}\right)_{4}$, tetraethyl ammonium hydroxide propagated smoothly in toluene at $85{ }^{\circ} \mathrm{C}$. To eliminate the possible terminal defect, the phenylboronic acid and bromobenzene were added successively to react with the bromo- and borate end groups, respectively. Finally, the polymers were collected by precipitating the reaction solution in an excess amount of methanol and the polymers were further purified by washing thoroughly with acetone by Soxhlet extraction.

The structures of the intermediates, monomers and polymers were characterized by ${ }^{1} \mathrm{H}$ and ${ }^{13} \mathrm{C}$ NMR spectra and satisfactory results were obtained (Fig. S1-S10, ESI $\dagger$ ). The resultant pTPE-DPA$\mathrm{Cz}$ and pTPE-DPA-Flu are soluble in commonly used organic solvents, such as tetrahydrofuran (THF), dichloromethane (DCM), chloroform, and toluene, which facilitate the solution process for PLED fabrication. The weight-average molecular weights of the resultant pTPE-DPA-Cz and pTPE-DPA-Flu were determined by advanced polymer chromatography (APC) in THF on the basis of a linear polystyrene calibration, and the values of $1.9 \times 10^{4}$ and $2.4 \times 10^{4} \mathrm{~g} \mathrm{~mol}^{-1}$ were obtained, respectively (Table S1, ESI $\dagger$ ), which are high enough for forming films with good quality. The resultant polymers are also thermally stable as revealed by the thermogravimetric analysis (TGA). The 5\% weight-loss temperatures $\left(T_{\mathrm{d}}\right)$ of pTPE-DPA-Cz and pTPE-DPAFlu are as high as 510 and $442{ }^{\circ} \mathrm{C}$, respectively (Fig. S11A, ESI $\dagger$ ). Moreover, their glass transition temperatures $\left(T_{\mathrm{g}}\right)$ are also as high as 193 and $152{ }^{\circ} \mathrm{C}$ as measured by differential scanning calorimetry (DSC) (Fig. S11B, ESI $\dagger$ ). These results indicate that our resultant polymers are stable enough for PLED applications as EMLs.

\section{Photophysical properties}

The UV-vis absorption and photoluminescence (PL) spectra of pTPE-DPA-Cz and pTPE-DPA-Flu in neat and doped films are shown in Fig. 1 and the data are summarized in Table 1. In THF solutions, THF/water mixtures with water fraction $\left(f_{\mathrm{w}}\right)$ of $99 \%$, neat films and doped films, pTPE-DPA-Cz and pTPE-DPA-Flu all show close absorption maxima at $c a .385 \mathrm{~nm}$. In THF solutions,
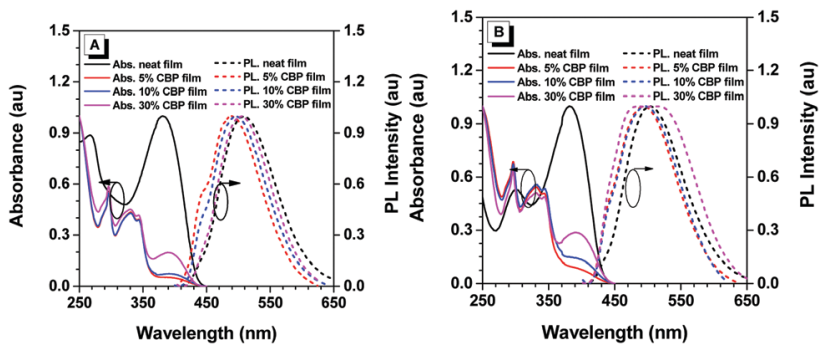

Fig. 1 The UV and photoluminescence (PL) spectra of (A) pTPE-DPA-Cz and (B) PTPE-DPA-Flu. The neat and doped films of polymers with weight ratios of 5,10 and $30 \%$ in CBP were deposited on a quartz substrates.

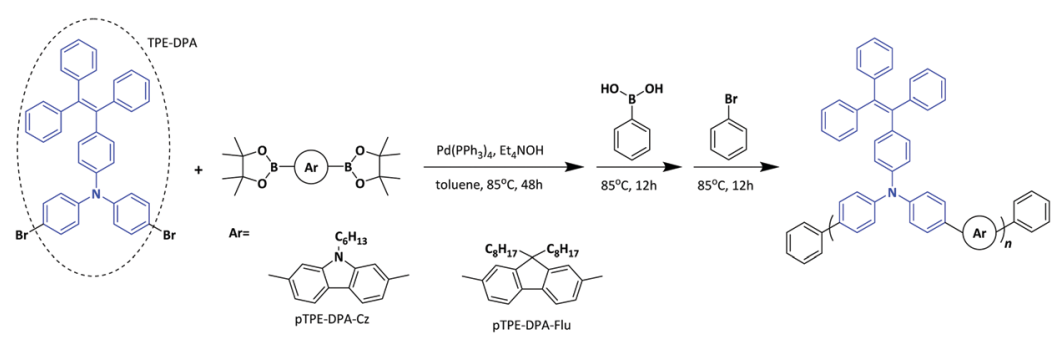

Scheme 1 Synthetic routes to PTPE-DPA-Cz and pTPE-DPA-Flu. 
Table 1 Photophysical properties of pTPE-DPA-Cz and pTPE-DPA-Flu

\begin{tabular}{|c|c|c|c|c|c|c|c|c|c|c|c|c|c|c|c|}
\hline & \multicolumn{3}{|l|}{$\operatorname{Soln}^{a}$} & \multicolumn{3}{|c|}{ Neat film ${ }^{b}$} & \multicolumn{9}{|c|}{ Doped film ${ }^{c}$} \\
\hline & $\lambda_{\mathrm{abs}}(\mathrm{nm})$ & $\lambda_{\mathrm{em}}(\mathrm{nm})$ & $\Phi_{\mathrm{F}}(\%)$ & $\lambda_{\mathrm{em}}(\mathrm{nm})$ & $\Phi_{\mathrm{F}}(\%)$ & $\tau(\mathrm{ns})$ & $\lambda_{\mathrm{em}}(\mathrm{nm})$ & $\Phi_{\mathrm{PL}}(\%)$ & $\tau(\mathrm{ns})$ & $\lambda_{\mathrm{em}}(\mathrm{nm})$ & $\Phi_{\mathrm{PL}}(\%)$ & $\tau(\mathrm{ns})$ & $\lambda_{\mathrm{em}}(\mathrm{nm})$ & $\Phi_{\mathrm{PL}}(\%)$ & $\tau(\mathrm{ns})$ \\
\hline pTPE-DPA-Cz & 383 & 500 & 0.4 & 508 & 28.7 & 2.5 & 486 & 58.3 & 2.9 & 496 & 63.3 & 3.0 & 503 & 50.8 & 2.9 \\
\hline
\end{tabular}

${ }^{a}$ Measured in THF solution $\left(2 \times 10^{-3} \mathrm{mg} \mathrm{mL}^{-1}\right)$ at room temperature. ${ }^{b}$ Deposited on a quartz substrate. ${ }^{c}$ In $\mathrm{CBP}$ matrix $(5 \mathrm{wt} \%, 10 \mathrm{wt} \%, 30 \mathrm{wt} \%$ respectively) deposited on quartz substrates.

pTPE-DPA-Cz and pTPE-DPA-Flu exhibit weak PL peaks at 500 and $495 \mathrm{~nm}$ respectively. While, with addition of the poor solvent of water into their THF solutions, the emission gradually intensified especially at high $f_{\mathrm{w}}$, demonstrating a typical AIE feature (Fig. 2). The PLQY measurement further confirms the AIE feature of these two polymers. The values of pTPE-DPA$\mathrm{Cz}$ and pTPE-DPA-Flu were recorded to be $0.4 \%$ and $1.5 \%$ in THF respectively, which reach up to $63.3 \%$ in their film states (Table 1).

Furthermore, compared with the neat films, the emission peaks of their doped films in a CBP host are blue-shifted slightly, which can be ascribed to the suppressed intermolecular dipole-dipole interaction and weakened intramolecular charge transfer effect from more polar neat films to less polar doped films, ${ }^{15}$ whereas, a slightly red-shifted emission of the doped film with $30 \mathrm{wt} \%$ pTPE-DPA-Flu might be attributed to the interaction between its functional groups and the host molecules. The room-temperature transient spectra of pTPE-DPA-Cz and pTPEDPA-Flu in neat and doped films were measured and the lifetimes of prompt fluorescence in the range from 2.3 to $3.0 \mathrm{~ns}$ were obtained, indicating that the polymers emit fluorescence (Table 1 and Fig. S12, ESI $\dagger$ ).

\section{Electrochemical properties}

The electrochemical properties of the pTPE-DPA-Cz and pTPEDPA-Flu were investigated using cyclic voltammetry (CV) and their electrochemical data are summarized in Table S1 (ESI $\dagger$ ). According to their scanned cyclic voltammograms (Fig. 3), the onset oxidation potential of pTPE-DPA-Cz and pTPE-DPA-Flu occurred at 0.76 and $0.69 \mathrm{~V}$, respectively. Based on the equation of $E_{\text {HOMO }}=-\left(E_{\text {onset(ox })}+4.8\right) \mathrm{eV}$ and the onset potential, the highest occupied molecular orbital (HOMO) energy levels of pTPE-DPA-Cz and pTPE-DPA-Flu were deduced

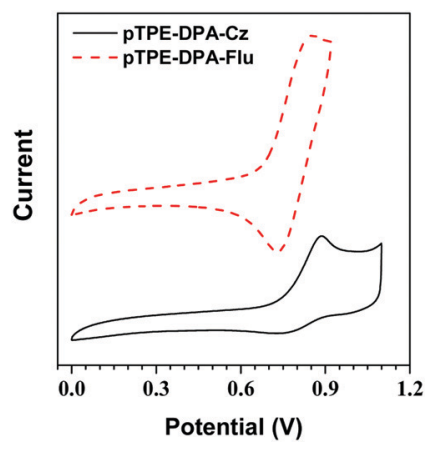

Fig. 3 Cyclic voltammograms of pTPE-DPA-Cz and pTPE-DPA-Flu measured in DCM containing $0.1 \mathrm{M}$ tetra- $n$-butylammonium hexafluorophosphate. Scan rate: $100 \mathrm{mV} \mathrm{s}^{-1}$.

to be $-\mathbf{5 . 5 6}$ and $-5.49 \mathrm{eV}$, respectively. As the onset reduction potential could not be observed clearly, the lowest unoccupied molecular orbital (LUMO) energy levels were alternatively calculated by the equation of $E_{\mathrm{LUMO}}=\left(E_{\mathrm{HOMO}}+E_{\mathrm{g}}^{\mathrm{opt}}\right) \mathrm{eV}$, where $E_{\mathrm{g}}^{\mathrm{opt}}$ was estimated by the absorption spectra of the polymer films. Therefore, the LUMO energy levels of the polymers were estimated to be -2.62 and $-2.57 \mathrm{eV}$.

\section{Theoretical calculations}

To gain deep insight into the photo-physical properties of the polymers, the distributions of the HOMOs and LUMOs, and the models of their repeating units of TPE-DPA-Cz and TPE-DPAFlu were calculated via density functional theory (DFT) at the B3LYP/6-31G(d) level. As illustrated in Fig. 4, the electron clouds of the HOMOs are located mainly on the triphenylamine units, whereas the electron clouds of the LUMOs are mostly localized on the TPE units, demonstrating that the fluorescence of the polymers mainly comes from the TPE-DPA units.
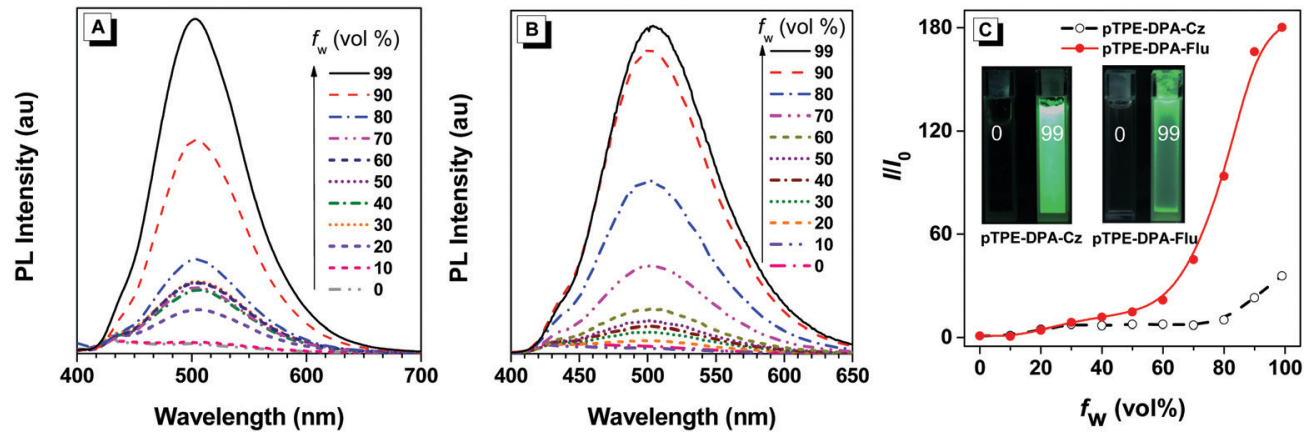

Fig. 2 PL spectra of (A) pTPE-DPA-Cz and (B) pTPE-DPA-Flu in THF/water mixtures with different water fractions $\left(f_{w}\right)$. (C) Plots of $I / I_{0}$ values versus $f_{w}$. $I_{0}$ is the PL intensity in pure THF. Inset: The photographs of the polymers in THF and THF/water mixtures with $f_{\mathrm{w}}$ of $99 \%$. 

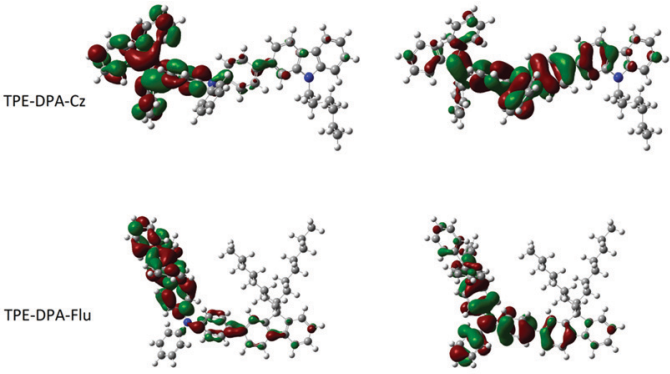

LUMO

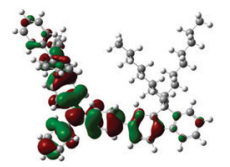

номо
Fig. 4 The HOMO and LUMO distributions of the calculation models of TPE-DPA-CZ and TPE-DPA-Flu obtained by DFT calculations performed at the B3LYP/6-31G(d) level.

\section{Film quality}

In consideration of the significant impact of film quality on the device performance, the quality and morphology of the spincoated films of these two AIE polymers were tested by atomic force microscopy (AFM). The films were prepared on silicon substrates by a spin-coating process which will be used for the device fabrication. As shown in Fig. 5, the neat films of pTPEDPA-Cz and pTPE-DPA-Flu exhibit smooth surfaces with small root mean square (RMS) values of 0.373 and $0.575 \mathrm{~nm}$, respectively, indicating that uniform films can be produced through a solution process. Similarly, their doped films also display small RMS values of 0.368 and $0.566 \mathrm{~nm}$. With the excellent film forming ability, the leakage current of the PLEDs could be greatly reduced, which benefits the injection of carriers and the uniformity of luminance.

\section{Electroluminescence}

To evaluate the EL performance of these two AIE polymers, we manufactured devices through a solution process with the configuration of ITO/PEDOT:PSS (50 nm)/EMLs/TmPyPB (40 nm)/ $\mathrm{LiF}(1 \mathrm{~nm}) / \mathrm{Al}$, in which indium tin oxide (ITO) acts as the anode,
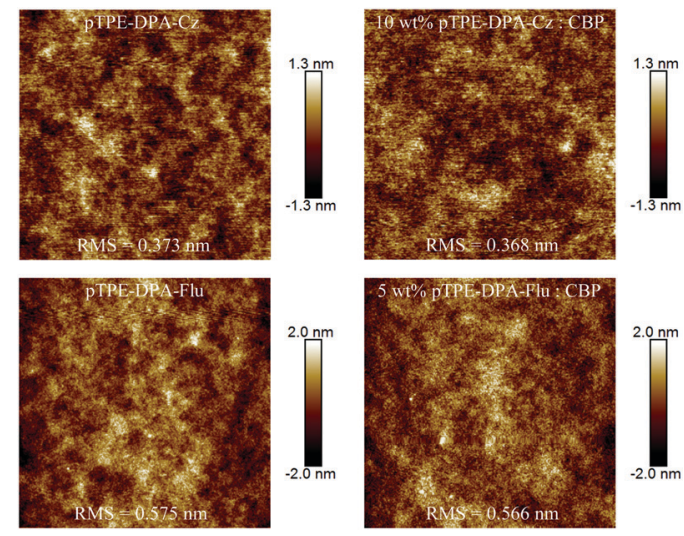

Fig. 5 AFM images of the neat and doped films of pTPE-DPA-Cz and pTPE-DPA-Flu. Neat films: Si/PEDOT:PSS (50 nm)/pTPE-DPA-Cz(60 nm); Si/PEDOT:PSS (50 nm)/pTPE-DPA-Flu(50 nm). Doped films: Si/PEDOT:PSS $(50 \mathrm{~nm}) / 10 \mathrm{wt} \%$ pTPE-DPA-Cz:CBP(55 nm); Si/PEDOT:PSS (50 nm)/5 wt\% pTPE-DPA-Flu:CBP(60 nm).

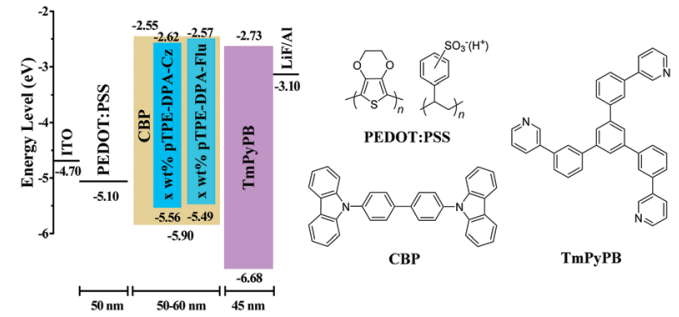

Fig. 6 Energy level diagram and molecular structures of the materials for PLEDs.

poly(3,4-ethylenedioxythiophene):poly(styrene-sulfonate) (PEDOT:PSS) and LiF are employed as hole- and electron-injecting layers, 1,3,5-tri( $m$-pyrid-3-yl-phenyl)benzene (TmPyPB) is selected as the electron-transporting layer, and $\mathrm{Al}$ is chosen for the cathode (Fig. 6). Devices 1-3 refer to the EMLs with 5, 10, and $30 \mathrm{wt} \%$ pTPE-DPA-Cz doped in CBP (55 nm), respectively, and device 4 for non-doped pTPE-DPA-Cz (60 nm) EML. Similarly, the devices 5-7 represent the EMLs of 5, 10, and $30 \mathrm{wt} \%$ pTPE-DPA-Flu doped in CBP $(60 \mathrm{~nm})$, respectively, and nondoped pTPE-DPA-Flu $(50 \mathrm{~nm})$ as the EML was assigned as device 8 .

The device performance data are listed in Table 2 and the curves are shown in Fig. 7 and Fig. S13 and S14 (ESI $\dagger$ ). For the devices 1-4, the EL peaks red shift from 474 to $517 \mathrm{~nm}$, suggesting that there might be intermolecular interactions. Among the doped devices, device 2 shows a maximum CE, power efficiency (PE), and EQE of $6.47 \mathrm{~cd} \mathrm{~A}^{-1}, 4.73 \mathrm{~lm} \mathrm{~W}^{-1}$, and $2.98 \%$, respectively. It is worth noting that the values could remain to be $6.02 \mathrm{~cd} \mathrm{~A}^{-1}, 4.53 \mathrm{~lm} \mathrm{~W}^{-1}$, and $2.77 \%$ at the luminance of $500 \mathrm{~cd} \mathrm{~m}^{-2}$, respectively, demonstrative of a low efficiency roll-off. Furthermore, the non-doped device 4 exhibits a maximum CE, PE, and EQE of $3.69 \mathrm{~cd} \mathrm{~A}^{-1}, 2.28 \mathrm{~lm} \mathrm{~W}^{-1}$ and $1.46 \%$, respectively. The same as the doped devices, a smaller efficiency roll-off is achieved with CE, PE and EQE values of $3.67 \mathrm{~cd} \mathrm{~A}^{-1}, 2.13 \mathrm{~lm} \mathrm{\textrm {W } ^ { - 1 }}$ and $1.44 \%$ at the luminance of $500 \mathrm{~cd} \mathrm{~m}^{-2}$, respectively. Quite similar behaviors were obtained for devices 5-8, and the efficiency roll-off of them is also very small. Therefore, it is feasible that highly efficient PLEDs with low efficiency roll-off can be fabricated by using AIE polymers as EMLs.

\section{Conclusions}

In summary, two AIE polymers, pTPE-DPA-Cz and pTPE-DPAFlu, bearing the AIE unit of TPE-DPA were designed and synthesized through Suzuki-Miyaura polycoupling. These two polymers possess good thermal stability and show high PLQY values in their solid and film states. Moreover, they also possess excellent film forming ability and can be fabricated into high quality films by a simple spin-coating process, which greatly benefits the device performance. Thus, doped and non-doped solution-processed PLEDs were fabricated using the AIE polymers as emitting layers. The results show that the doped device of pTPE-DPA-Flu with 5\% doping concentration in the CBP host 
Table 2 EL performances of PLEDs based on PTPE-DPA-Cz (devices 1-4) and pTPE-DPA-Flu (devices 5-8)

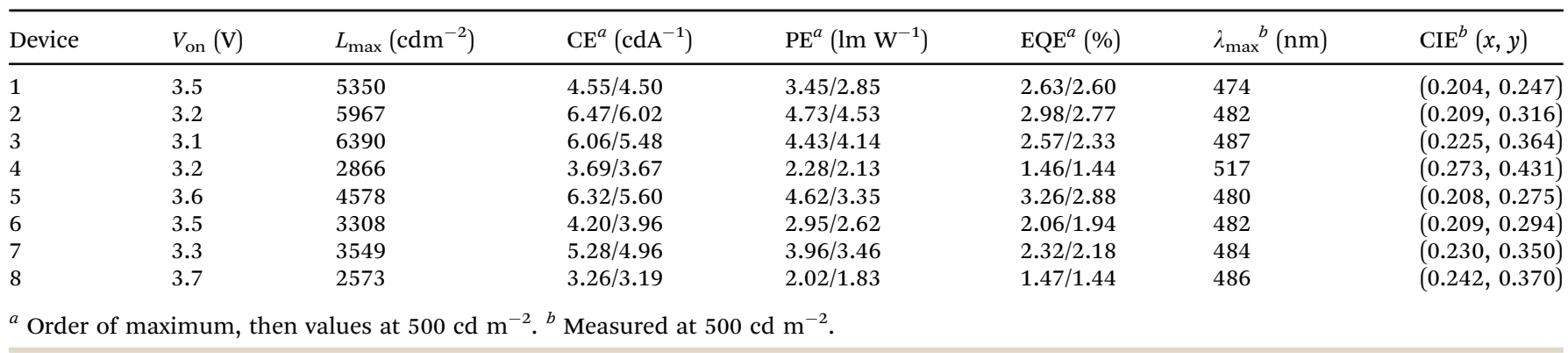
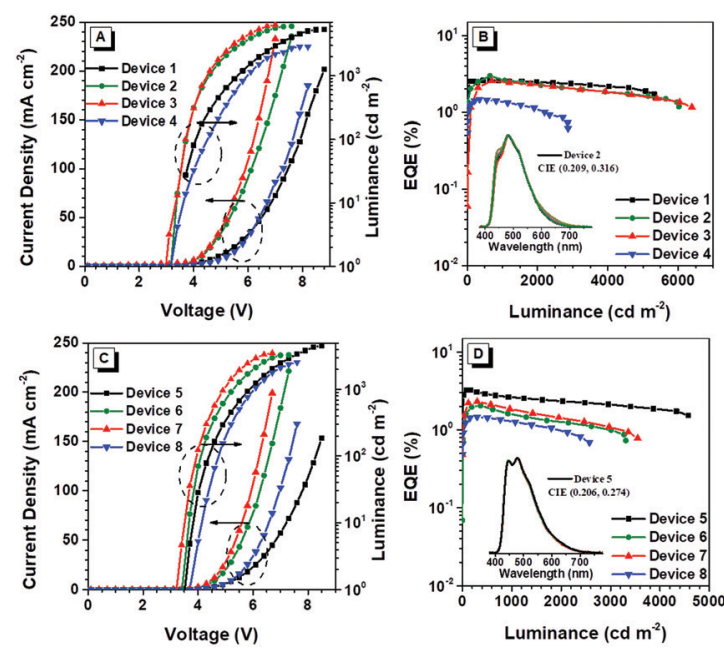

Fig. 7 (A and C) Current density-luminance-voltage curves of devices 1-8. (B) and (D) External quantum efficiency as a function of luminance for devices 1-8. Inset: Normalized EL spectra of devices 2 (B) and 5 (D).

exhibits a maximum EQE of $3.26 \%$ and the non-doped PLED using pTPE-DPA-Cz as EML could perform with CE as high as $3.69 \mathrm{~cd} \mathrm{~A}^{-1}$. More importantly, unlike most PLEDs based on ACQ polymers, which suffer from serious efficiency roll-off, these PLEDs show quite low efficiency roll-off, demonstrating their outstanding efficiency stability. These results indicate that the AIE polymers can be ideal candidates for the construction of high-performance solution-processed PLEDs with low efficiency roll-off.

\section{Conflicts of interest}

There are no conflicts to declare.

\section{Acknowledgements}

This work was financially supported by the National Natural Science Foundation of China (21788102 and 21525417), the Natural Science Fund of Guangdong Province (2019B030301003 and 2016A030312002), and the Innovation and Technology Commission of Hong Kong (ITC-CNERC14SC01).

\section{References}

1 C. W. Tang and S. A. Vanslyke, Organic electroluminescent diodes, Appl. Phys. Lett., 1987, 51, 913.

2 (a) H. Wu, L. Ying, W. Yang and Y. Cao, Progress and perspective of polymer white light-emitting devices and materials, Chem. Soc. Rev., 2009, 38, 3391; (b) C. Sekine, Y. Tsubata, T. Yamada, M. Kitano and S. Doi, Recent progress of high performance polymer OLED and OPV materials for organic printed electronics, Sci. Technol. Adv. Mater., 2014, 15, 034203; (c) F. Xua, H. U. Kima, J.-H. Kima, B. J. Jung, A. C. Grimsdale and D.-H. Hwang, Progress and perspective of iridium-containing phosphorescent polymers for light-emitting diodes, Prog. Polym. Sci., 2015, 47, 92.

3 (a) C. A. Zuniga, S. Barlow and S. R. Marder, Approaches to Solution-Processed Multilayer Organic Light-Emitting Diodes Based on Cross-Linking, Chem. Mater., 2011, 23, 658; (b) S. Shao, J. Ding, L. Wang, X. Jing and F. Wang, Highly Efficient Blue Electrophosphorescent Polymers with Fluorinated Poly(arylene ether phosphine oxide) as Backbone, J. Am. Chem. Soc., 2012, 134, 15189; (c) S. Gong, C. Yang and J. Qin, Efficient phosphorescent polymer light-emitting diodes by suppressing triplet energy back transfer, Chem. Soc. Rev., 2012, 41, 4797.

4 J. H. Burroughes, D. D. C. Bradley, A. R. Brown, R. N. Marks, K. Mackay, R. H. Friend, R. L. Burns and A. B. Holmes, Light-emitting diodes based on conjugated polymers, Nature, 1990, 347, 539.

5 (a) J. Luo, G. Xie, S. Gong, T. Chen and C. Yang, Creating a thermally activated delayed fluorescence channel in a single polymer system to enhance exciton utilization efficiency for bluish-green electroluminescence, Chem. Commun., 2016, 52, 2292; (b) Z. Ren, R. S. Nobuyasu, F. B. Dias, A. P. Monkman, S. Yan and M. R. Bryce, Pendant Homopolymer and Copolymers as Solution-Processable Thermally Activated Delayed Fluorescence Materials for Organic Light-Emitting Diodes, Macromolecules, 2016, 49, 5452; (c) G. Xie, J. Luo, M. Huang, T. Chen, K. Wu, S. Gong and C. Yang, Inheriting the Characteristics of TADF Small Molecule by Side-Chain Engineering Strategy to Enable Bluish Green Polymers with High PLQYs up to 74\% and External Quantum Efficiency over 16\% in Light-Emitting Diodes, Adv. Mater., 2017, 29, 1604223; (d) Y. Hu, W. Cai, D. Chen, L. Ying, X. Yang, X. Jiang, S. Su, F. Huang and Y. Cao, Novel efficient blue and bluish-green 
light emitting polymers with delayed fluorescence, J. Mater. Chem. C, 2018, 6, 2690; (e) Y. Yang, S. Wang, Y. Zhu, Y. Wang, H. Zhan and Y. Cheng, Thermally Activated Delayed Fluorescence Conjugated Polymers with Backbone-Donor/PendantAcceptor Architecture for Nondoped OLEDs with High External Quantum Efficiency and Low Roll-Off, Adv. Funct. Mater., 2018, 28, 1706916.

6 S. Shao, J. Hu, X. Wang, L. Wang, X. Jing and F. Wang, Blue Thermally Activated Delayed Fluorescence Polymers with Nonconjugated Backbone and Through-Space Charge Transfer Effect, J. Am. Chem. Soc., 2017, 139, 17739.

7 (a) Z. Xu, J. Gu, X. Qiao, A. Qin, B. Z. Tang and D. Ma, Highly Efficient Deep Blue Aggregation-Induced Emission Organic Molecule: A Promising Multifunctional Electroluminescence Material for Blue/Green/Orange/Red/White OLEDs with Superior Efficiency and Low Roll-Off, ACS Photonics, 2019, 6, 767; (b) Z. Xu, J. Gu, J. Huang, C. Li, Y. Li, D. Yang, X. Qiao, A. Qin, Z. Zhao, B. Z. Tang and D. Ma, Design and performance study of high efficiency/low efficiency roll-off/high CRI hybrid WOLEDs based on aggregation-induced emission materials as fluorescent emitters, Mater. Chem. Front., 2019, 3, 2652.

8 (a) M. Chen, L. Li, H. Nie, J. Tong, L. Yan, B. Xu, J. Z. Sun, W. Tian, Z. Zhao, A. Qin and B. Z. Tang, Tetraphenylpyrazinebased AIEgens: facile preparation and tunable light emission, Chem. Sci., 2015, 6, 1932; (b) F. Song, Z. Xu, Q. Zhang, Z. Zhao, H. Zhang, W. Zhao, Z. Qiu, C. Qi, H. Zhang, H. H. Y. Sung, I. D. Williams, J. W. Y. Lam, Z. Zhao, A. Qin, D. Ma and B. Z. Tang, Highly Efficient Circularly Polarized Electroluminescence from Aggregation-Induced Emission Luminogens with Amplified Chirality and Delayed Fluorescence, Adv. Funct. Mater., 2018, 28, 1800051; (c) Z. Qiu, B. Hao, X. Gu, Z. Wang, N. Xie, J. W. Y. Lam, H. Hao and B. Z. Tang, A general powder dusting method for latent fingerprint development based on AIEgens, Sci. China: Chem., 2018, 61, 966.

9 (a) J. Liu, J. W. Y. Lam and B. Z. Tang, Aggregation-induced Emission of Silole Molecules and Polymers: Fundamental and Applications, J. Inorg. Organomet. Polym., 2009, 19, 249; (b) R. Hu, A. Qin and B. Z. Tang, AIE polymers: Synthesis and applications, Prog. Polym. Sci., 2020, 100, 101176.
10 J. W. Y. Lam, J. Chen, C. C. W. Law, H. Peng, Z. Xie, K. K. L. Cheuk, H. S. Kwok and B. Z. Tang, Silolecontaining linear and hyperbranched polymers: synthesis, thermal stability, light emission, nano-dimensional aggregation, and optical power limiting, Macromol. Symp., 2003, 196, 289.

11 R. Hu, X. Chen, T. Zhou, H. Si, B. He, R. T. K. Kwok, A. Qin and B. Z. Tang, Lab-in-cell based on spontaneous amino-yne click polymerization, Sci. China: Chem., 2019, 62, 1198.

12 (a) W. Wu, S. Ye, R. Tang, L. Huang, Q. Li, G. Yu, Y. Liu, J. Qin and Z. Li, New tetraphenylethylene-containing conjugated polymers: Facile synthesis, aggregation-induced emission enhanced characteristics and application as explosive chemsensors and PLEDs, Polymer, 2012, 53, 3163; (b) W. Wu, S. Ye, L. Huang, X. Li, Y. Fu, Q. Huang, G. Yu, Y. Liu, J. Qin, Q. Li and Z. Li, A conjugated hyperbranched polymer constructed from carbazole and tetraphenylethylene moieties: convenient synthesis through one-pot "A2 + B4" Suzuki polymerization, aggregation-induced enhanced emission, and application as explosive chemosensors and PLEDs, J. Mater. Chem., 2012, 22, 6374; (c) W. Wu, S. Ye, G. Yu, Y. Liu, J. Qin and Z. Li, Novel Functional Conjugative Hyperbranched Polymers with Aggregation-Induced Emission: Synthesis Through One-Pot “A2 + B4" Polymerization and Application as Explosive Chemsensors and PLEDs, Macromol. Rapid Commun., 2012, 33, 164.

13 Y. Xie, Y. Gong, M. Han, F. Zhang, Q. Peng, G. Xie and Z. Li, Tetraphenylcyclopentadiene-Based Hyperbranched Polymers: Convenient Syntheses from One Pot “A4 + B2” Polymerization and High External Quantum Yields up to $9.74 \%$ in OLED Devices, Macromolecules, 2019, 52, 896.

14 R. Hu, N. L. Leung and B. Z. Tang, AIE macromolecules: syntheses, structures and functionalities, Chem. Soc. Rev., 2014, 43, 4494.

15 G. Méhes, K. Goushi, W. J. Potscavage and C. Adachi, Influence of host matrix on thermally-activated delayed fluorescence: Effects on emission lifetime, photoluminescence quantum yield, and device performance, Org. Electron., 2014, 15, 2027. 